\title{
Changes in hemodynamic parameters with the use of etomidate versus ketamine induction in the emergency department
}

\author{
Abdullah Bakhsh ${ }^{1, *}$, Maryam Alnashri ${ }^{2}$, Fatimah Alawami ${ }^{2}$, Rafal Aseel ${ }^{2}$, \\ Maha Almaghthawi ${ }^{2}$, Ghaida Alrahaili ${ }^{2}$, Anas Bifari ${ }^{2}$, Hassan Algethami ${ }^{2}$
}

${ }^{1}$ Department of Emergency Medicine, the King Abdulaziz University, 80215 Jeddah, Saudi Arabia

${ }^{2}$ Faculty of Medicine, the King Abdulaziz University, 80215 Jeddah, Saudi Arabia

*Correspondence

aarbakhsh@kau.edu.sa

(Abdullah Bakhsh)

\begin{abstract}
Objectives: Etomidate is the sedative agent of choice during rapid sequence intubation (RSI) owing to its hemodynamic stability, rapid onset of action, and short duration of action. Nevertheless, ketamine is rapidly gaining popularity as an alternative agent, primarily because of its catecholamine-mediated effects. This feature has prompted clinicians to use ketamine for hemodynamically unstable patients. The aim of this study was to compare the percent change in hemodynamic parameters resulting from the use of etomidate versus ketamine during RSI in the emergency department.

Methods: This cross-sectional prospective observational study conducted at an academic emergency department included patients recruited from March 2018 through May 2019 on a convenience basis when the principal investigator was scheduled to work in the emergency department.

Results: Our study showed a percent reduction in all hemodynamic parameters with the use of ketamine: $-13.14 \%$ in systolic blood pressure, $-10.40 \%$ in diastolic blood pressure, $-10.15 \%$ in mean arterial pressure, and $-1.12 \%$ in heart rate. Moreover, the rate of $\geq 20 \%$ reduction in hemodynamic parameters with ketamine was $27.27 \%$ in systolic blood pressure, $18.18 \%$ in diastolic blood pressure, $18.18 \%$ in mean arterial pressure, and $27.27 \%$ in heart rate.

Conclusions: Although ketamine has a sympathomimetic effect, it may cause hemodynamic instability in select patients. Therefore, caution is advised when using ketamine routinely during RSI, especially in critically ill patients in the emergency department.
\end{abstract}

\section{Keywords}

Rapid sequence induction and intubation; Ketamine; Etomidate; Hemodynamic response; Acute reduction in hemodynamic parameters; Catecholamine depletion

\section{Introduction}

Rapid sequence intubation (RSI) is the cornerstone of emergency airway management [1]. The administration of a sedative agent immediately followed by a neuromuscular blocking agent to produce rapid unconsciousness and paralysis provides optimum intubation conditions while minimizing aspiration $[2,3]$. Nevertheless, selecting an appropriate sedative agent is a critical decision in emergency medicine. The ideal sedative agent is rapid acting, has a short duration of action, allows optimum intubation conditions, and is reliably effective and safe [4]. Hemodynamic control is also crucial for critically ill patients requiring RSI in the emergency department. Therefore, emergency physicians must be aware of the hemodynamic effects produced by the medications used during intubation.

Etomidate is a sedative agent popular among emergency physicians. It is a sedative-hypnotic with a very stable hemo- dynamic profile and a reliably rapid onset of action (10-15 s) and duration of action (4-10 min) when administered intravenously at a dose of $0.3 \mathrm{mg} / \mathrm{kg}$. It inhibits excitatory stimuli by enhancing the effect of gamma-aminobutyric acid [4, 5]. A prospective observational study published in 2000 by Smith et $a l$. investigated the hemodynamic effects of etomidate after RSI and found no significant change in blood pressure or heart rate in their patients [6]. Another prospective observational study published in 2006 by Zed et al. evaluated the hemodynamic effects of etomidate during RSI and found that the mean baseline systolic blood pressure, diastolic blood pressure, and heart rate were $132 \pm 35.4 \mathrm{mmHg}, 69 \pm 21.2 \mathrm{mmHg}$, and $96 \pm$ 26.2 beats per minute, respectively. They reported an overall elevation of $1.5 \mathrm{mmHg}$ in systolic blood pressure $(P=0.0254)$ and $0.5 \mathrm{mmHg}$ in diastolic blood pressure $(P=0.2213)$ at 10 min after intubation [7]. In contrast, a randomized controlled trial conducted in the operating room on patients scheduled 
for elective surgery showed a decrease in the mean systolic blood pressure from $133 \pm 14 \mathrm{mmHg}$ to $117 \pm 9 \mathrm{mmHg}$ and mean diastolic blood pressure from $78 \pm 11 \mathrm{mmHg}$ to $72 \pm 10$ $\mathrm{mmHg}$ [8].

Ketamine has also been gaining popularity among emergency physicians because of its catecholamine-mediated maintenance of blood pressure. Nonetheless, the rapid surge in the use of ketamine has revealed unpredictable hemodynamic effects during RSI. Early research in critically ill patients found ketamine to be occasionally associated with a reduction in the mean arterial pressure and cardiac output [9-11]. Recent clinical studies on RSI with ketamine reported hypotension rates of $3.6 \%$ to $24 \%$, which may be harmful, especially in patients with an initial systolic blood pressure less than 100 $\mathrm{mmHg}$ [12]. A prospective observational study conducted on critically ill patients in the pre-hospital stage undergoing RSI with ketamine showed a blunted hypertensive response and more frequent hypotension in patients with a high shock index $(>0.9)$ [12].

Studies comparing patient-centered outcomes between etomidate and ketamine show no difference in morbidity and mortality $[13,14]$. However, few studies have directly compared the hemodynamic responses to etomidate and ketamine. The clinical question of whether ketamine should be used routinely for RSI deserves investigation. We believe such a study would provide significant insights on the hemodynamic response to ketamine induction. Therefore, the primary objective of this study was to compare the change in hemodynamic parameters between two commonly available induction agents, namely etomidate and ketamine. The secondary objective was to compare the rate of acute reduction in hemodynamic parameters between etomidate and ketamine. We hypothesized that ketamine would demonstrate a percent elevation in hemodynamic parameters when compared to that of etomidate.

\section{Methods}

Ethics approval for the study was obtained from the unit of biomedical ethics (reference 692-18). A waiver of consent was granted because of the observational and emergency nature of the study.

\subsection{Study design and setting}

This was a cross-sectional prospective observational study conducted at an academic emergency department with approximately 62,000 annual visits. Patients were enrolled on a convenience basis when the principal investigator was working in the department. The study period was from March 2018 through May 2019. All adult patients undergoing RSI were enrolled. The airway management team comprised an emergency medicine resident, a registered nurse, a respiratory therapist, and a board-certified emergency physician. Patients were intubated by emergency medicine residents under the supervision of a board-certified emergency physician. All emergency medicine residents undergo an annual emergency airway bootcamp. Additionally, all medical providers are required to maintain certification in advanced cardiac life support and advanced trauma life support. The department also has an
RSI guideline in place (Supplementary 1). Data were collected in real time using a standardized intubation procedure note (Supplementary 2). The note included patient information, diagnosis, indication for intubation, pre- and post-intubation vital signs, pre- and post-intubation drugs (with dose and time), number of intubation attempts, device used, operator level, and complications. Vital signs were documented within $10 \mathrm{~min}$ before and after medication administration. The intubation procedure note is completed by both the physician performing the procedure and the nurse assisting with the procedure. Medications and intubation methods were determined by the treating physician and were not influenced by this study.

\subsection{Definition}

RSI was defined as the administration of a sedative agent followed by a neuromuscular blocking agent.

\subsection{Study protocol}

All patients undergoing RSI were screened by the principal investigator for inclusion. Treatment decisions and medication selection were made at the discretion of the treating clinician and were not influenced by the study. Indications for RSI included hypoxic respiratory failure, hypercapnic respiratory failure, airway protection, and anticipated clinical course. Prior to induction, the patient's position was optimized, a complete set of vital signs were measured and documented, and all necessary equipment were prepared and made available at the bedside. RSI medications were pre-prepared in labeled syringes and induction was achieved by administering a predetermined dose based on the patient's estimated weight (etomidate: $0.3 \mathrm{mg} / \mathrm{kg}$ intravenous; ketamine: $1-2 \mathrm{mg} / \mathrm{kg}$ intravenous). Following induction and paralysis, the trachea was intubated with an endotracheal tube. Correct placement was confirmed clinically and via quantitative waveform capnography. Procedure details were documented on a standardized intubation procedure note $10 \mathrm{~min}$ before induction and $10 \mathrm{~min}$ after induction. The selection of the 10-min time point before and after induction was based on expert consensus within the department. The principal investigator was present during every intubation to ensure protocol adherence and accurate documentation.

\subsection{Selection of participants}

We included patients aged $\geq 18$ years old who underwent RSI by emergency physicians for 1) respiratory distress, 2) hypoxic respiratory failure, 3) hypercapnic respiratory failure, 4) airway protection, or 5) anticipated clinical course. We excluded patients aged $<18$ years, pregnant women, patients receiving push-dose pressors, and patients not undergoing RSI.

\subsection{Monitoring}

All patients underwent continuous noninvasive monitoring of heart rate, respiratory rate, oxygen saturation, blood pressure, and capnography using CARESCAPE B650 (GE Healthcare, Chicago, IL, USA). A blood pressure cuff was placed on one upper extremity and measurements were recorded $10 \mathrm{~min}$ before intubation and $10 \mathrm{~min}$ after intubation. The average of 
three blood pressure readings was recorded.

\subsection{Outcome measures}

The primary outcome was the percent reduction in hemodynamic parameters in response to etomidate versus ketamine. The secondary outcome was the incidence of acute reduction ( $\geq 20 \%$ reduction and any reduction) in hemodynamic parameters in response to etomidate versus ketamine.

\subsection{Data collection and statistical analysis}

The treating clinician and registered nurse assisting with the procedure completed a standardized intubation procedure note in real time at $10 \mathrm{~min}$ before induction and $10 \mathrm{~min}$ after induction. Thereafter, the content was entered into an Excel worksheet. Content was crosschecked by the study investigators, including an experienced board-certified emergency physician. Data were analyzed using a data analysis toolkit in Microsoft Excel for Office 365 MSO (16.0.12527.21294) 32bit (Microsoft, Redmond, WA, USA). Categorical data were reported as frequencies and percentages. Percent changes in hemodynamic parameters were calculated using the following formula: [(pre-hemodynamic parameter - post-hemodynamic parameter)/pre-hemodynamic parameter $\times 100]$. The chisquare test was used to compare categorical data, and the $t$ test (two-sample assuming unequal variances) was used to compare numerical data. Statistical significance was set at a two-tailed $P$-value $<0.05$.

TA B L E 1. Patient characteristics, indication for intubation and mean dose of induction agents.

\begin{tabular}{|lc|}
\hline Age \pm SD & $56.64 \pm 17.89$ \\
\hline Gender & \\
\hline Male, $\mathbf{n}(\%)$ & $24(49.00 \%)$ \\
\hline Indication for intubation, $\mathbf{n}(\%)$ & \\
\hline Airway protection & $22(44.80 \%)$ \\
\hline Hypoxic respiratory failure & $11(22.40 \%)$ \\
\hline Hypercapnic respiratory failure & $4(8.20 \%)$ \\
\hline Anticipated clinical course & $2(4.10 \%)$ \\
\hline Mean dose in milligrams $( \pm$ SD) & \\
\hline Etomidate & $22.55 \mathrm{mg}( \pm 4.79)$ \\
\hline Ketamine & $90.00 \mathrm{mg}( \pm 7.26)$ \\
\hline
\end{tabular}

\section{Results}

During the 13-month study period, 119 patients in the emergency department were screened. Among them, 70 patients were excluded. Among the remaining 49 patients, 38 received etomidate for induction, while 11 received ketamine. The mean age of the patients was $56.64 \pm 17.89$ (SD) years, and $49.00 \%$ were male. The mean etomidate dose was $22.55 \pm$ 4.79 (SD) $\mathrm{mg}$, whereas the mean ketamine dose was 90.00 \pm 7.26 (SD) $\mathrm{mg}$. The primary indications for intubation included airway protection $(44.8 \%)$, hypoxic respiratory failure (22.4\%), hypercapnic respiratory failure $(8.2 \%)$, and antici- pated clinical course (4.1\%) (Table 1).

Etomidate was the most used induction agent in $77.55 \%$ of the patients, while ketamine was used in only $22.44 \%$ of the patients. The overall first-pass success rate in this sample was $77.55 \%$. First-pass success rate was $76.31 \%$ in the etomidate group and $72.72 \%$ in the ketamine group. Overall, good glottic views were observed in $71.43 \%$ of the patients, including $78.94 \%$ in the etomidate group but only $45.45 \%$ in the ketamine group $(P=0.76)$. Fentanyl was used in $71.05 \%$ of the patients in the etomidate group and $81.81 \%$ in the ketamine group. Succinylcholine was used in $89.47 \%$ of the patients in the etomidate group and $81.81 \%$ in the ketamine group (Table 2).

\subsection{Hemodynamic response}

The primary outcome of percent reduction in hemodynamic parameters was not statistically different between the etomidate and ketamine groups (Table 3). Patients receiving etomidate showed a negative reduction in all hemodynamic parameters: $-15.02 \%$ in systolic blood pressure, $-20.25 \%$ in diastolic blood pressure, $-19.33 \%$ in mean arterial pressure, and $-2.24 \%$ in heart rate. Similarly, patients receiving ketamine showed a negative reduction in all hemodynamic parameters: $-13.14 \%$ in systolic blood pressure, $-10.40 \%$ in diastolic blood pressure, $-10.15 \%$ in mean arterial pressure, and $-1.12 \%$ in heart rate (Table 4).

The rate of $\geq 20 \%$ reduction in hemodynamic parameters was statistically similar between the two groups (Table 5). The rates in the etomidate versus those in the ketamine group were as follows: $26.32 \%$ vs. $27.27 \%$ (odds ratio [OR] $=1.05$; $95 \%$ confidence interval $[\mathrm{CI}]=0.32-4.74)$ for systolic blood pressure, $31.58 \%$ vs. $18.18 \%(\mathrm{OR}=0.48 ; 95 \% \mathrm{CI}=0.11-2.57)$ for diastolic blood pressure, $28.94 \%$ vs. $18.18 \%(\mathrm{OR}=0.45$; $95 \% \mathrm{CI}=0.12-2.94)$ for mean arterial pressure, and $7.89 \% \mathrm{vs}$. $27.27 \%(\mathrm{OR}=4.37 ; 95 \% \mathrm{CI}=0.87-25.81)$ for heart rate.

The rate of any reduction in hemodynamic parameters was also statistically similar between the two groups (Table 5). The rates of reduction in the etomidate versus those in the ketamine group were as follows: $57.89 \%$ vs. $63.63 \%(\mathrm{OR}=1.27 ; 95 \%$ $\mathrm{CI}=0.47-5.09)$ for systolic blood pressure, $57.89 \% \mathrm{vs} .45 .45 \%$ $(\mathrm{OR}=0.60 ; 95 \% \mathrm{CI}=0.23-2.33)$ for diastolic blood pressure, $57.89 \%$ vs. $54.54 \%(\mathrm{OR}=0.87 ; 95 \% \mathrm{CI}=0.34-3.36)$ for mean arterial pressure, and $44.74 \%$ vs. $36.36 \%(\mathrm{OR}=0.70 ; 95 \% \mathrm{CI}$ $=0.26-2.81$ ) for heart rate.

Fig. 1,2,3,4 show graphical illustrations of the absolute changes in the mean hemodynamic parameters between the etomidate and ketamine groups.

\section{Discussion}

Previous studies directly comparing the hemodynamic effects of ketamine with those of etomidate for RSI in the emergency department setting are scant. The pharmacologic and safety profiles of etomidate offer many advantages for RSI in the emergency department setting [4, 7]. While etomidate remains the gold standard for induction in RSI, ketamine has unpredictable effects on hemodynamic parameters based on the patient's catecholamine stores [7]. A dose-related increase 
TA B L E 2. Frequency of induction agent, first pass success rates and good glottic views, pretreatment use, and paralytic use (based on induction agent).

\begin{tabular}{|lcccc} 
& Overall, n (\%) & Etomidate, $\mathbf{n}(\%)$ & Ketamine, n (\%) & $P$ value \\
\hline Use & $49(100.00 \%)$ & $38(77.55 \%)$ & $11(22.44 \%)$ & \\
\hline First pass success & $38(77.55 \%)$ & $29(76.31 \%)$ & $8(72.72 \%)$ & $\mathbf{0 . 9 7}$ \\
\hline Good glottic view (CL grade 1-2) & $35(71.43 \%)$ & $30(78.94 \%)$ & $5(45.45 \%)$ & $\mathbf{0 . 7 6}$ \\
\hline Fentanyl & $36(73.46 \%)$ & $27(71.05 \%)$ & $9(81.81 \%)$ & $\mathbf{0 . 9 3}$ \\
\hline Succinylcholine & $43(87.75 \%)$ & $34(89.47 \%)$ & $9(81.81 \%)$ & $\mathbf{0 . 9 5}$ \\
Rocuronium & $6(12.24 \%)$ & $4(10.53 \%)$ & $2(18.18 \%)$ & $\mathbf{0 . 8 8}$ \\
\hline Video laryngoscopy & $42(85.71 \%)$ & $34(89.47 \%)$ & $7(63.63 \%)$ & $\mathbf{0 . 8 3}$ \\
\hline
\end{tabular}

CL: Cormack-Lehane.

TA B L E 3. Mean hemodynamic parameters 10-minutes pre-induction and 10-minutes post-induction with percent changes.

\begin{tabular}{|c|c|c|c|c|}
\hline \multicolumn{4}{|c|}{ Etomidate } & Percent change $(\%)$ \\
\hline Pre-SBP $(\mathbf{m m H g} \pm \mathrm{SD})$ & $137.68 \pm 25.31$ & Post-SBP (mmHg \pm SD) & $128.63 \pm 37.67$ & $-15.02 \%$ \\
\hline Pre-DBP $(\mathrm{mmHg} \pm \mathrm{SD})$ & $81 \pm 16.03$ & Post-DBP $(\mathrm{mmHg} \pm \mathrm{SD})$ & $73.34 \pm 22.42$ & $-20.45 \%$ \\
\hline Pre-MAP $(\mathrm{mmHg} \pm \mathrm{SD})$ & $101.67 \pm 21.50$ & Post-MAP $(\mathrm{mmHg} \pm \mathrm{SD})$ & $91.78 \pm 26.73$ & $-19.33 \%$ \\
\hline Pre-HR (bpm \pm SD) & $111.13 \pm 26.93$ & Post-HR (bpm \pm SD) & $109.76 \pm 23.05$ & $-2.24 \%$ \\
\hline \multicolumn{4}{|c|}{ Ketamine } & ent change $(\%)$ \\
\hline Pre-SBP $(\mathrm{mmHg} \pm \mathrm{SD})$ & $148.72 \pm 56.06$ & Post-SBP $(\mathrm{mmHg} \pm \mathrm{SD})$ & $131.72 \pm 36.59$ & $-13.14 \%$ \\
\hline Pre-DBP $(\mathrm{mmHg} \pm \mathrm{SD})$ & $79.27 \pm 20.37$ & Post-DBP (mmHg \pm SD) & $72.18 \pm 13.62$ & $-10.40 \%$ \\
\hline Pre-MAP $(\mathrm{mmHg} \pm \mathrm{SD})$ & $102.36 \pm 31.04$ & Post-MAP $(\mathrm{mmHg} \pm \mathrm{SD})$ & $93.20 \pm 19.48$ & $-10.15 \%$ \\
\hline Pre-HR (bpm \pm SD) & $118.63 \pm 29.55$ & Post-HR (bpm \pm SD) & $124.27 \pm 45.31$ & $-1.12 \%$ \\
\hline
\end{tabular}

SBP: systolic blood pressure, DBP: diastolic blood pressure, MAP: mean arterial pressure, HR: heart rate.

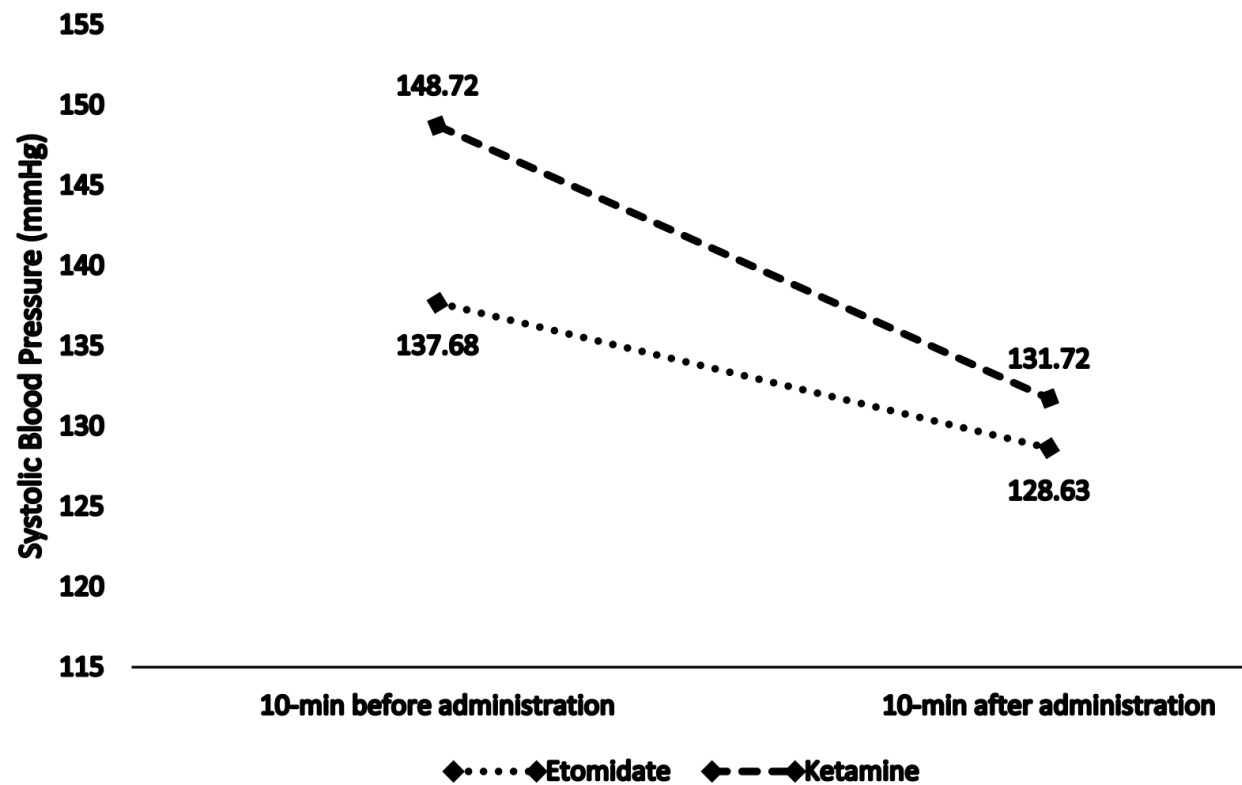

F I G U RE 1. Mean systolic blood pressure 10-minutes before and after induction.

in hemodynamics is seen in patients who are not catecholamine depleted. However, patients who are catecholamine depleted and lack autonomic control experience direct myocardial depressant effects, leading to dose-dependent negative ionotropic and chronotropic effects [7]. Nevertheless, our study revealed a statistically similar percent reduction in all hemodynamic parameters when comparing etomidate with ketamine. Importantly, ketamine showed a greater mean percent reduction in all hemodynamic parameters. This is supported by a study by Waxman et al. [11], who investigated 12 critically ill 


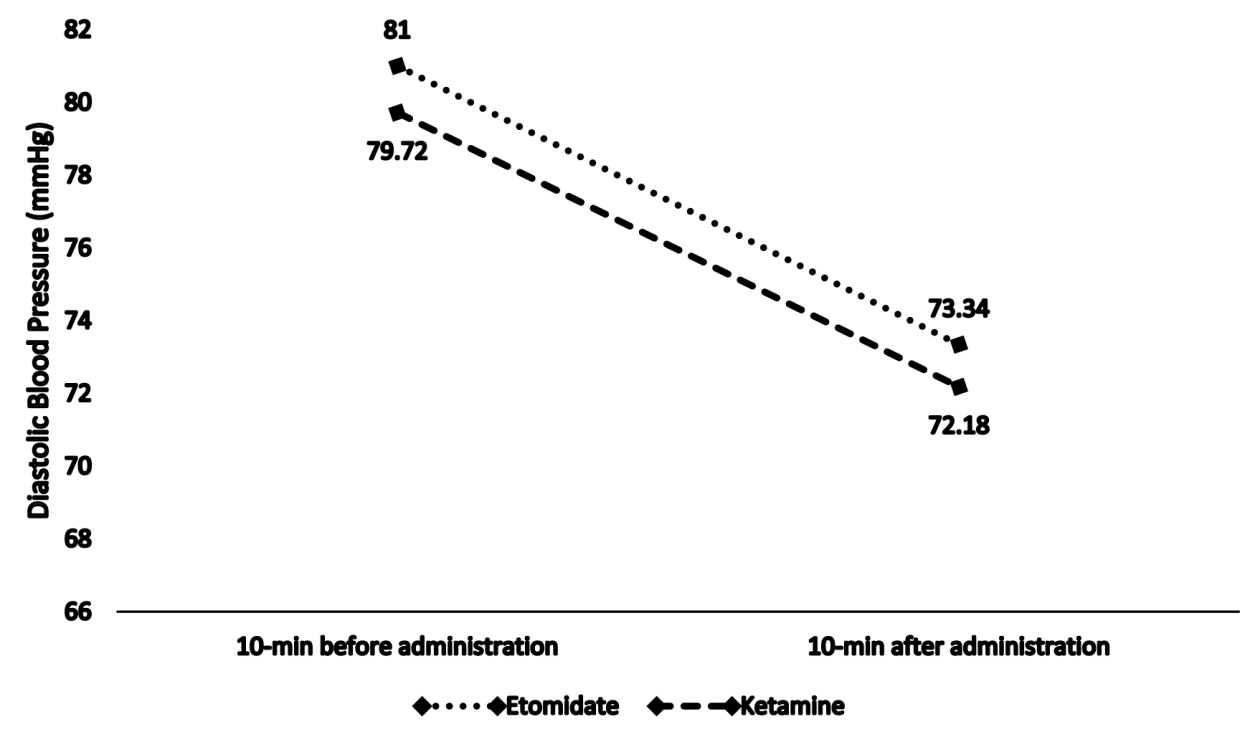

F I G U R E 2. Mean diastolic blood pressure 10-minutes before and after induction.

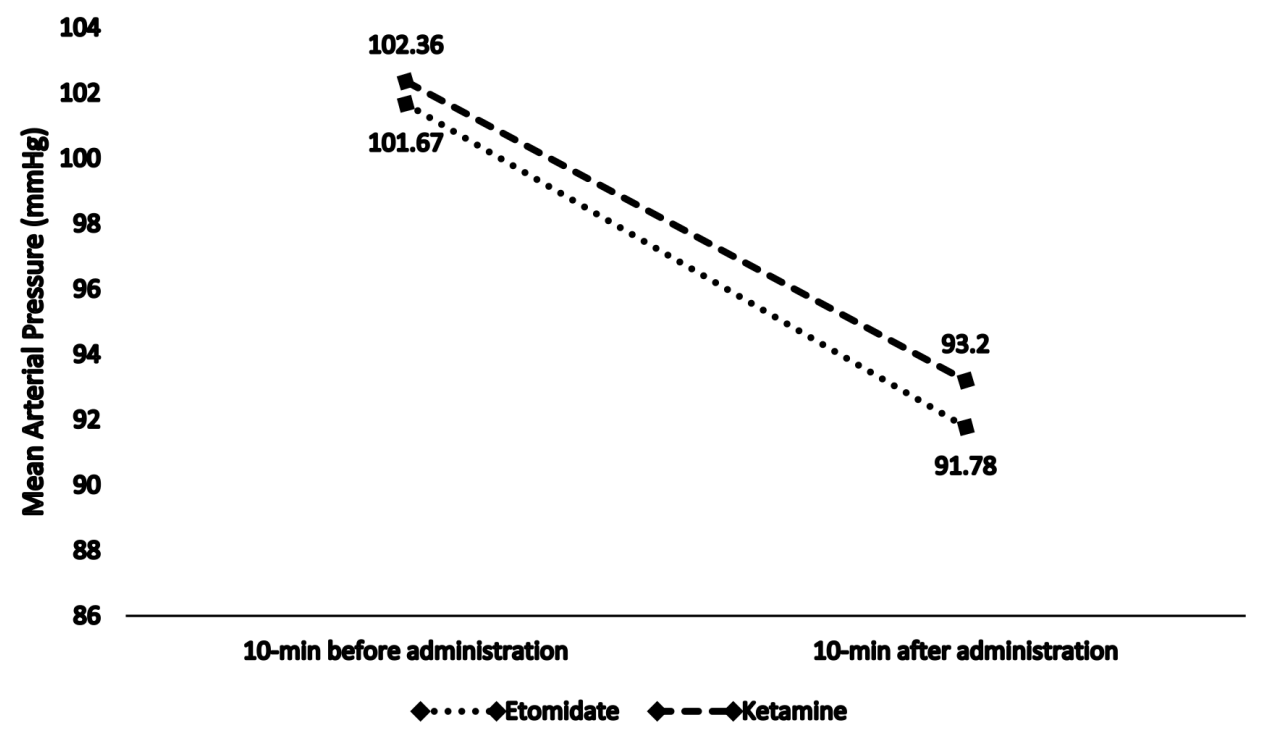

F I G U R E 3. Mean arterial pressure 10-minutes before and after induction.

TA B L E 4. Comparison of mean percent reduction in hemodynamic parameters between etomidate and ketamine.

\begin{tabular}{|lccc|} 
& Etomidate & Ketamine & $\boldsymbol{P}$-value \\
\hline SBP & $-15.02 \%$ & $-13.14 \%$ & $\mathbf{0 . 8 6 6}$ \\
\hline DBP & $-20.45 \%$ & $-10.40 \%$ & $\mathbf{0 . 3 4 0}$ \\
\hline MAP & $-19.33 \%$ & $-10.15 \%$ & $\mathbf{0 . 3 9 8}$ \\
HR & $-2.24 \%$ & $-1.12 \%$ & $\mathbf{0 . 8 7 5}$ \\
\hline
\end{tabular}

SBP: systolic blood pressure, DBP: diastolic blood pressure, MAP: mean arterial pressure, HR: heart rate.

patients by comparing hemodynamic variables pre-ketamine and 5 min post-ketamine-only induction. In that study, one patient developed severe bradycardia, four demonstrated decreased cardiac output and mean arterial pressure, and six had decreased ventricular contractility. Lipmann et al. [15] repeated these measurements at 2-min intervals up to $15 \mathrm{~min}$ after induction in 22 critically ill patients. They reported that although myocardial performance was improved in the majority of patients, the response was not uniform, and patients with hypovolemia or those with prolonged operative stress were at most risk of reduced cardiac output. In vitro, ketamine is negatively ionotropic when applied directly to the cardiac muscle [16]. In vivo, however, it acts by releasing centrally mediated catecholamines and inhibiting their reuptake. This mechanism accounts for the hemodynamic stability of ketamine [17, 18]. Alternatively, post-intubation hypotension may not necessarily be an effect of pharmacology but may result from an interaction between the hypovolemic state of the patient, onset of positivepressure ventilation, and a subsequent reduction in venous return $[19,20]$.

Moreover, the incidence of acute reduction in all hemodynamic parameters was statistically similar. When comparing our study to previous ones that investigated similar hemodynamic parameters in the context of ketamine use in either a pre- 
T A B L E 5. Incidence of acute reduction in hemodynamic parameter between etomidate and ketamine.

\begin{tabular}{|c|c|c|c|}
\hline \multicolumn{4}{|c|}{ Acute reduction in hemodynamic parameters ( $\geq 20 \%$ reduction) } \\
\hline & Etomidate & Ketamine & Odds ratio $[95 \% \mathrm{CI}]$ \\
\hline SBP & $26.32 \%$ & $27.27 \%$ & $1.05[0.32-4.75]$ \\
\hline DBP & $31.58 \%$ & $18.18 \%$ & $0.48[0.11-2.57]$ \\
\hline MAP & $28.94 \%$ & $18.18 \%$ & $0.45[0.12-2.94]$ \\
\hline HR & $7.89 \%$ & $27.27 \%$ & $4.37[0.87-25.81]$ \\
\hline \multicolumn{4}{|c|}{ Acute reduction in hemodynamic parameters (any reduction) } \\
\hline & Etomidate & Ketamine & Odds ratio $[95 \% \mathrm{CI}]$ \\
\hline SBP & $57.89 \%$ & $63.63 \%$ & $1.27[0.47-5.09]$ \\
\hline DBP & $57.89 \%$ & $45.45 \%$ & $0.60[0.23-2.33]$ \\
\hline MAP & $57.89 \%$ & $54.54 \%$ & $0.87[0.34-3.36]$ \\
\hline HR & $44.74 \%$ & $36.36 \%$ & $0.70[0.26-2.81]$ \\
\hline
\end{tabular}

SBP: systolic blood pressure, DBP: diastolic blood pressure, MAP: mean arterial pressure, $H R$ : heart rate.

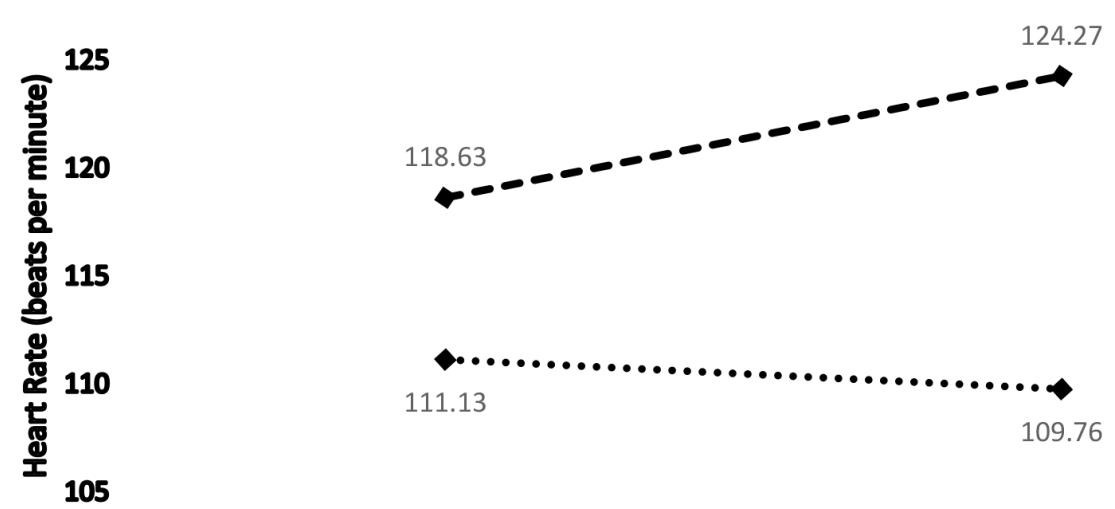

100

10-min before administration

10-min after administration

$\diamond . . . \downarrow$ Etomidate $\bullet-\neg$ Ketamine

F I G U R E 4. Mean heart rate 10-minutes before and after induction.

hospital or emergency setting, we found similar results with some differences that may be explained by various factors, such as different sample sizes and definitions of endpoints. Price et al. [20] conducted a retrospective study that compared the hemodynamic effects of etomidate to those of ketamine in 100 patients undergoing endotracheal intubation (ETI) in a pre-hospital setting. Researchers assessed up to two sets of vital signs before ETI and up to five sets of vital signs after ETI. The median pre-ketamine heart rate was $99-100$ beats per minute and the median post-ketamine heart rate was 92101 beats per minute. The median pre-ketamine systolic blood pressure was $128-140 \mathrm{mmHg}$ and the median post-ketamine systolic blood pressure was 123-137 mmHg. Furthermore, the rate of occurrence of bradycardia in their study was $9.3 \%$ and that of hypotension was $36.1 \%$ [20]. We observed a greater percent reduction in hemodynamic parameters, with a rate of $\geq 20 \%$ reduction in heart rate of $27.27 \%$ with ketamine and the rate of any reduction in heart rate of $63.63 \%$. In contrast, the rates of $\geq 20 \%$ reduction in systolic blood pressure, diastolic blood pressure, and mean arterial pressure with ketamine were $27.27 \%, 18.18 \%$, and $18.18 \%$, respectively. The rates of any reduction in systolic blood pressure, diastolic blood pressure, and mean arterial pressure were $63.63 \%, 45.45 \%$, and $54.54 \%$, respectively. The different results reported in our study may be attributed to differences in the definition of events. Ballow et al. [21] compared the outcomes of 177 patients who received ketamine for RSI to those of 266 patients who received etomidate and reported hypotension in only $3.6 \%$ of the patients in the ketamine group compared to $6 \%$ in the etomidate group. Their study, despite the reasonable sample size, reported significantly lower hypotensive events than did our study. It appears that hemodynamic events after ketamine administration depend on many factors that include, but are not limited to, patient factors and perhaps ketamine doses. 
In summary, we can clearly state that despite ketamine's sympathomimetic effect, it may cause hemodynamic instability in select patients. Ketamine increases the central nervous system sympathetic outflow and decreases the reuptake of catecholamines, producing an elevation in hemodynamic parameters. On the other hand, it can also cause cardiac depression after induction. This effect is commonly overlooked in clinical practice. Therefore, patients who are apparently catecholamine depleted and lack autonomic control will experience direct myocardial depressant effects, leading to negative ionotropic and chronotropic effects [22,23]. This may explain why we observed a reduction in all hemodynamic parameters comparable to those of etomidate.

This study also has some limitations. It was an observational study with a small convenience-based sample and lacked randomization. Additionally, we relied on noninvasive blood pressure monitoring, which may yield inaccurate results [21]. However, invasive blood pressure monitoring is not practical for every patient undergoing RSI. Moreover, many patients had incomplete data, and they could not be analyzed. Furthermore, we did not account for other factors affecting hemodynamic changes, including but not limited to pretreatment agent use, type of airway device used, volume/cardiac status prior to induction, and amount of positive-pressure ventilation.

\section{Conclusions}

The use of ketamine as an induction agent during RSI was associated with a reduction in all hemodynamic parameters when compared to those of etomidate. Therefore, we advise caution with the use of ketamine during RSI, especially for select patients in the emergency department. Ketamine may not necessarily improve hemodynamic parameters in critically ill catecholamine-depleted patients. A randomized clinical trial assessing these induction agents in hemodynamically compromised patients would undoubtedly help inform clinical practice.

\section{ETHICS APPROVAL AND CONSENT TO PARTICIPATE}

Ethics approval for the study was obtained from the unit of biomedical ethics (reference 692-18). A waiver of consent was granted because of the observational and emergency nature of the study.

\section{AUTHOR CONTRIBUTIONS}

Abdullah Bakhsh designed the study, collected data in real-time, assisted with statistical analysis and drafted the manuscript. Maryam Alnashri, Fatimah Alawami, Rafal Aseel, Maha Almaghthawi, Ghada Alrahaili, Anas Bifari, and Hassan Algethami assisted with data collection, performed statistical analysis, analyzed the results, and assisted with drafting the manuscript.

\section{ACKNOWLEDGMENT}

We would like to thank the emergency department staff at the King Abdulaziz University Hospital for their diligent work in providing care for critically ill patients. We also would like to thank the Research Summer School at the King Abdulaziz University for their continuous support during the project.

\section{FUNDING}

This research study received no financial support.

\section{CONFLICT OF INTEREST}

The authors of this study declare no conflicts of interest.

\section{DATA AVAILABILITY}

Data to support findings are available from the corresponding author upon request.

\section{SUPPLEMENTARY MATERIAL}

Supplementary material associated with this article can be found, in the online version, at https://oss.signavitae. com/mre-signavitae/article/1348925342553653248/ attachment/Supplementary\%20material.rar.

\section{REFERENCES}

[1] Dibble C. Rapid sequence induction in the emergency department by emergency medicine personnel. Emergency Medicine Journal. 2006; 23 : 62-64.

[2] Okubo M, Gibo K, Hagiwara Y, Nakayama Y, Hasegawa K. The effectiveness of rapid sequence intubation (RSI) versus non-RSI in emergency department: an analysis of multicenter prospective observational study. International Journal of Emergency Medicine. 2017; 10: 1.

[3] Reynolds SF, Heffner J. Airway management of the critically ill patient. Chest. 2005; 127: 1397-1412.

[4] Hampton JP. Rapid-sequence intubation and the role of the emergency department pharmacist. American Journal of Health-System Pharmacy. 2011; 68: 1320-1330.

[5] Scherzer D, Leder M, Tobias JD. Pro-con debate: etomidate or ketamine for rapid sequence intubation in pediatric patients. The Journal of Pediatric Pharmacology and Therapeutics. 2012; 17: 142-149.

[6] Smith DC, Bergen JM, Smithline H, Kirschner R. A trial of etomidate for rapid sequence intubation in the emergency department. The Journal of Emergency Medicine. 2000; 18: 13-16.

[7] Zed PJ, Abu-Laban RB, Harrison DW. Intubating conditions and hemodynamic effects of etomidate for rapid sequence intubation in the emergency department: an observational cohort study. Academic Emergency Medicine. 2006; 13: 378-383.

[8] Shah S, Chowdhury I, Bhargava A, Sabbharwal B. Comparison of hemodynamic effects of intravenous etomidate versus propofol during induction and intubation using entropy guided hypnosis levels. Journal of Anaesthesiology Clinical Pharmacology. 2015; 31: 180.

[9] Wong DHW, Jenkins LC. The cardiovascular effects of ketamine in hypotensive states. Canadian Anaesthetists' Society Journal. 1975; 22: 339-348.

[10] Weiskopf R, Bogetz M, Roizen M, Reid I. Cardiovascular and metabolic sequelae of inducing anesthesia with ketamine or thiopental in hypovolemic swine. Anesthesiology. 1984; 60: 214-219.

[11] Waxman K, Shoemaker WC, Lippmann M. Cardiovascular effects of 
anesthetic induction with ketamine. Anesthesia \& Analgesia. 1980; 59: 355-358.

[12] Miller M, Kruit N, Heldreich C, Ware S, Habig K, Reid C, et al. Hemodynamic response after rapid sequence induction with ketamine in out-of-hospital patients at risk of shock as defined by the shock index. Annals of Emergency Medicine. 2016; 68: 181-188.e2.

[13] Jabre P, Combes X, Ricard-Hibon A, Mirat P, Cibien J, Bourzeix C, et al. Etomidate versus ketamine for rapid sequence intubation in acutely ill patients: a multicenter randomized controlled trial. Critical Care. 2009; 13: P405.

[14] Upchurch CP, Grijalva CG, Russ S, Collins SP, Semler MW, Rice TW, et al. Comparison of etomidate and ketamine for induction during rapid sequence intubation of adult trauma patients. Annals of Emergency Medicine. 2017; 69: 24-33.e2.

[15] Lippmann M, Appel PL, Mok MS, Shoemaker WC. Sequential cardiorespiratory patterns of anesthetic induction with ketamine in critically ill patients. Critical Care Medicine. 1983; 11: 730-734.

[16] Gelissen HPMM, Epema AH, Henning RH, Krijnen HJ, Hennis PJ, den Hertog A. Inotropic effects of propofol, thiopental, midazolam, etomidate, and ketamine on isolated human atrial muscle. Anesthesiology. 1996; 84: 397-403.

[17] Morris C, Perris A, Klein J, Mahoney P. Anaesthesia in haemodynamically compromised emergency patients: does ketamine represent the best choice of induction agent? Anaesthesia. 2009; 64: 532-539.

[18] Shafi S, Gentilello L. Pre-hospital endotracheal intubation and positive pressure ventilation is associated with hypotension and decreased survival in hypovolemic trauma patients: an analysis of the national trauma data bank. Journal of Trauma and Acute Care Surgery. 2005; 59: 1140-1147.

[19] Franklin C, Samuel J, Hu T. Life-threatening hypotension associated with emergency intubation and the initiation of mechanical ventilation. The American Journal of Emergency Medicine. 1994; 12: 425-428.

[20] Price B, Arthur AO, Brunko M, Frantz P, Dickson JO, Judge T, et al. Hemodynamic consequences of ketamine vs etomidate for endotracheal intubation in the air medical setting. The American Journal of Emergency Medicine. 2013; 31: 1124-1132.

[21] Ballow SL, Kaups KL, Anderson S, Chang M. A standardized rapid sequence intubation protocol facilitates airway management in critically injured patients. Journal of Trauma and Acute Care Surgery. 2012; 73: 1401-1405.

[22] Schwartz DA, Horwitz LD. Effects of ketamine on left ventricular performance. Survey of Anesthesiology. 1975; 194: 410-414.

[23] Traber DL, Wilson RD, Priano LL. Differentiation of the cardiovascular effects of CI-581. Anesthesia \& Analgesia. 1968; 47: 769-778.

How to cite this article: Abdullah Bakhsh, Maryam Alnashri, Fatimah Alawami, Rafal Aseel, Maha Almaghthawi, Ghada Alrahaili, et al. Changes in hemodynamic parameters with the use of etomidate versus ketamine induction in the emergency department. Signa Vitae. 2021;17(2):85-92. doi:10.22514/sv.2021.005. 